\title{
Circulating CTRP1 Levels in Type 2 Diabetes and Their Association with FGF21
}

\author{
Sora Han, ${ }^{1}$ Jong Dai Kim, ${ }^{2}$ Sunyi Lee, ${ }^{1}$ Ae Lee Jeong, ${ }^{1}$ Jeong Su Park, ${ }^{1}$ Hyo Jeong Yong, ${ }^{1}$ \\ Ariundavaa Boldbaatar, ${ }^{1}$ Hye In Ka, ${ }^{1}$ Eun-Jung Rhee, ${ }^{3}$ Won-Young Lee, ${ }^{3}$ and Young Yang ${ }^{1}$ \\ ${ }^{1}$ Department of Biological Sciences, Sookmyung Women's University, 04310 Seoul, Republic of Korea \\ ${ }^{2}$ Department of Internal Medicine, Konyang University Buyeo Hospital, Buyeo, Republic of Korea \\ ${ }^{3}$ Division of Endocrinology and Metabolism, Department of Internal Medicine, Sungkyunkwan University School of Medicine, \\ Kangbuk Samsung Hospital, Seoul, Republic of Korea
}

Correspondence should be addressed to Won-Young Lee; wonyoung2.lee@samsung.com and Young Yang; yyang@sm.ac.kr

Received 26 January 2016; Revised 26 April 2016; Accepted 4 May 2016

Academic Editor: Javier Salvador

Copyright (C) 2016 Sora Han et al. This is an open access article distributed under the Creative Commons Attribution License, which permits unrestricted use, distribution, and reproduction in any medium, provided the original work is properly cited.

\begin{abstract}
The goal of this study was to investigate whether circulating Clq/TNF- $\alpha$-related protein 1 (CTRP1) levels are associated with diabetes. In addition, relationships between CTRP1 and other diabetes-related cytokines were elucidated, including adiponectin and fibroblast growth factor 21 (FGF21). A total of 178 subjects (78 men and 100 women) aged 29-70 years (mean age, 46.1 years) were randomly selected. The sera from a normal glucose tolerance group $(n=68)$ and a prediabetes/type 2 diabetes group $(n=110)$ were collected; then, circulating levels of CTRP1, adiponectin, and FGF21 were determined via enzyme-linked immunosorbent assay in all sera. Subjects with either prediabetes or diabetes exhibited higher circulating CTRP1 levels than healthy subjects. Sera analysis revealed that CTRP1 was positively correlated with age, body mass index, fasting blood glucose, and circulating FGF21 levels. However, CTRP1 was negatively correlated with total cholesterol and total circulating adiponectin levels in univariate analysis. In addition, multivariate analysis found that CTRP1 was independently associated with age, fasting blood glucose, and circulating FGF21 levels. CTRP1 was correlated with homeostasis model assessment- $\beta$ (HOMA- $\beta$ ), but no correlation was observed with HOMA-insulin resistance. In conclusion, circulating CTRP1 levels are increased in subjects with type 2 diabetes and are positively associated with circulating FGF21 levels.
\end{abstract}

\section{Introduction}

Glucose metabolism is systemically regulated by the endocrine system. In addition to insulin and glucagon, a variety of secreted proteins including adiponectin, fibroblast growth factor 21 (FGF21), and Clq/TNF- $\alpha$-related protein 1 (CTRP1) influence each other to regulate glucose homeostasis. Abnormal production and secretion of those hormones result in defects in glucose metabolism by causing insulin resistance (IR) in the livers, skeletal muscles, and adipose tissues, which leads to type 2 diabetes mellitus (T2DM) [1-3]. Thus, controlling the concentration or activity of these newly elucidated hormones is regarded as an alternative therapeutic approach to improve insulin sensitivity in T2DM [4-6].

CTRP1 has recently been found to be a novel metabolic hormone [7]. CTRP1 shares its structural homology with adiponectin [8] and has been identified as a novel adipokine that is ubiquitously expressed, unlike adiponectin [7, 9]. Although the specific CTRP1 receptor has not yet been elucidated, studies with rodents show that administration of CTRP1 is sufficient to lower blood glucose levels and increase energy expenditure by activating the AMPK signaling cascade in the skeletal muscles [10]. Clinical studies show that circulating CTRP1 levels are positively correlated with fasting blood glucose levels and homeostatic model assessmentinsulin resistance (HOMA-IR) in Chinese patients and nonalcoholic fatty liver patients [11-14]. It was also reported that circulating CTRP1 levels are higher in subjects with metabolic syndrome than those in healthy subjects [15]. However, it remains unclear whether the functional role of increased CTRP1 is to enhance or to aggravate insulin sensitivity, 
although CTRP1 is known to increase fatty acid oxidation and oxygen consumption in animal studies $[12,14,16]$.

In this clinical study, circulating CTRP1 levels were measured in Korean subjects with normal glucose tolerance, prediabetes (preDM), and T2DM. Furthermore, levels of circulating adiponectin and FGF21 which are responsible for maintaining glucose homeostasis were measured to assess the relationship between those secreted proteins and CTRP1.

\section{Materials and Methods}

2.1. Study Subject. Healthy subjects were recruited from a population of patients who visited the Health Screening Center at Kangbuk Samsung Hospital in 2003. In total, 178 subjects who participated in an annual health checkup were randomly selected for this study. Subjects with viral hepatitis, other liver diseases, chronic renal disease, hypertension, excessive alcohol consumption (>20 g/day), or fasting blood glucose higher than $100 \mathrm{mg} / \mathrm{dL}$ were excluded [17]. Subjects with preDM consisted of the Kangbuk Samsung HospitalDiabetes Prevention Project cohort, who had undergone comprehensive health examination and subsequently underwent an oral glucose tolerance test (OGTT). These subjects agreed to join a cohort study at the Kangbuk Samsung Hospital from December 2011 to July 2013. After a routine health examination, subjects who had shown a fasting blood glucose equal to or greater than $100 \mathrm{mg} / \mathrm{dL}$ were referred to the outpatient clinic in the endocrinology department. Then, they underwent a standard $2 \mathrm{~h} 75 \mathrm{~g}$ OGTT. PreDM was diagnosed when fasting blood glucose levels were between 100 and $126 \mathrm{mg} / \mathrm{dL}$ and $2 \mathrm{~h}$ levels were between 140 and $200 \mathrm{mg} / \mathrm{dL}$. T2DM was diagnosed when fasting blood glucose levels were higher than $126 \mathrm{mg} / \mathrm{dL}$ or HbAlc was higher than $6.5 \%$. This study protocol was approved by the Institutional Review Board and the Ethics Committee of Kangbuk Samsung Hospital and was carried out according to the principles of the 1975 Declaration of Helsinki. Written informed consent was provided by all subjects.

2.2. Laboratory Examinations. All sera were obtained in the morning, following $12-14 \mathrm{~h}$ of fasting. Sera were distributed to tubes and stored at $-80^{\circ} \mathrm{C}$ for further experiments. Circulating levels of fatty acid-binding protein 4 (FABP4), monocyte chemoattractant protein 1 (MCP1), retinol-binding protein 4 (RBP4), interleukin-6 (IL-6), visfatin, tumor necrosis factor$\alpha$ (TNF- $\alpha$ ), CTRP1 (all from Biovendor, Brno, Czech Republic), FGF21, and total adiponectin (all from Alpco Diagnostics; Salem, NH, USA) were measured using an enzymelinked immunosorbent assay (ELISA) according to the manufacturer's protocols. Blood glucose, total cholesterol, triglycerides (TGs), high-density lipoprotein cholesterol (HDLC), and low-density lipoprotein cholesterol (LDL-C) were measured as previously described [18]. To estimate insulin sensitivity and $\beta$-cell function, HOMA was used, which is based on fasting blood glucose and insulin levels [19].

2.3. Statistical Analyses. All data are presented as the mean \pm SD or proportion except where otherwise indicated. The following tests were used to compare the clinical and laboratory parameters according to circulating CTRP1 level tertile: oneway analysis of variance, Tukey's post hoc test, and the linearby-linear association test. Pearson's and Kendall's tau-b correlation coefficients were calculated to evaluate the associations between circulating CTRP1 levels and clinical/laboratory measurements. Multiple regression analyses were performed to find an independent factor for CTRP1. Age, sex, body mass index (BMI), total cholesterol, systolic/diastolic blood pressure, fasting blood glucose, triglycerides, FGF21, adiponectin, and medications (sulphonylurea, DPP-IV inhibitor, alphaglucosidase inhibitor, insulin, ACE inhibitor, ARB, thiazide, statin, fibrate, thioctic acid, and cilostazol) are included in this model and all variables controlled each other. All statistical analyses were performed using a SPSS version 18.0 (Chicago, IL, USA). $P$ values $<0.05$ were considered statistically significant.

\section{Results}

3.1. Baseline Characteristics of Subjects with PreDM and T2DM. The average age of the 178 individual study subjects was $46.1 \pm 12.0$ years, and their average BMI was $23.9 \pm$ $3.1 \mathrm{~kg} / \mathrm{m}^{2}$. Study subjects were categorized as healthy, preDM, or T2DM, according to fasting blood glucose level, OGTT, and $\mathrm{HbAlc}$ level. The levels of fasting blood glucose, insulin, $\mathrm{HbA1c}$, and circulating FGF21 were significantly increased in subjects with preDM and T2DM, compared to healthy subjects. However, unlike other parameters, circulating adiponectin levels were negatively correlated with the fasting blood glucose level (Table 1).

3.2. Relationship between Circulating CTRP1 Levels and T2DM. It was observed that the population showing high circulating CTRP1 level (over $300 \mathrm{ng} / \mathrm{mL}$ ) was more distributed in preDM or T2DM groups than normal group (Figure 1(a)). Additionally, circulating CTRP1 levels were significantly increased in both subjects with preDM $(352.8 \pm 327.9 \mathrm{ng} / \mathrm{mL})$ and T2DM $(355.3 \pm 341.3 \mathrm{ng} / \mathrm{mL})$, compared with normal subjects $(103.7 \pm 53.4 \mathrm{ng} / \mathrm{mL})$ on average. However, there was no statistical significance between circulating CTRP1 levels in subjects with preDM and T2DM (Figure 1(b)). As expected and previously reported $[12,14]$, circulating CTRP1 levels were positively correlated with fasting blood glucose levels (Figure 1(c)). Even when BMI, age, sex, and medications were controlled, CTRP1 independently and positively correlated with fasting blood glucose levels (Table 2). On the other hand, circulating CTRP1 levels were significantly and negatively correlated with HOMA- $\beta$ (Figure $1(\mathrm{~d})$ ), while there was no correlation between CTRP1 and HOMA-IR (Table 3).

3.3. The Association of CTRP1 with Other Clinical and Laboratory Parameters. Next, Pearson's correlation analysis was performed to assess the relationship between CTRP1 and other clinical and laboratory parameters in study subjects. As shown in Table 4, circulating CTRP1 levels were positively correlated with BMI, fasting blood glucose, and TG levels, whereas they were negatively correlated with total cholesterol and circulating adiponectin levels in univariate analysis. 
TABLE 1: Baseline characteristics of study participants.

\begin{tabular}{|c|c|c|c|c|c|}
\hline & $\begin{array}{l}\text { Normal } \\
(n=68)\end{array}$ & $\begin{array}{l}\text { PreDM } \\
(n=52)\end{array}$ & $\begin{array}{c}\mathrm{DM} \\
(n=58)\end{array}$ & $\begin{array}{c}P \text { value } \\
\text { (normal versus } \\
\text { PreDM) }\end{array}$ & $\begin{array}{c}P \text { value } \\
\text { (PreDM } \\
\text { versus } \\
\text { DM) }\end{array}$ \\
\hline BMI $\left(\mathrm{kg} / \mathrm{m}^{2}\right)$ & $22.45 \pm 2.7$ & $24.28 \pm 2.9$ & $25.3 \pm 3.1$ & 0.001 & 0.097 \\
\hline SBP (mmHg) & $106.6 \pm 11.4$ & $115.2 \pm 16.0$ & $117.9 \pm 11.1$ & 0.002 & 0.410 \\
\hline Glucose (mg/dL) & $90.0 \pm 5.5$ & $108.8 \pm 6.3$ & $141.8 \pm 32.1$ & 0.000 & 0.000 \\
\hline Insulin (mg/dL) & $7.4 \pm 2.1$ & $10.0 \pm 4.9$ & $12.0 \pm 7.2$ & 0.000 & 0.083 \\
\hline HOMA-IR & $1.6 \pm 0.5$ & $2.7 \pm 1.5$ & $4.4 \pm 3.3$ & 0.000 & 0.000 \\
\hline HOMR- $\beta$ & $103.6 \pm 37.0$ & $79.3 \pm 35.3$ & $60.2 \pm 32.7$ & 0.000 & 0.011 \\
\hline HbAlc (\%) & No data & $5.8 \pm 0.3$ & $7.2 \pm 0.9$ & NA & 0.000 \\
\hline Adiponectin (ng/mL) & $4427.0 \pm 2043.6$ & $2760.7 \pm 2112.3$ & $1483.3 \pm 1135.6$ & 0.000 & 0.001 \\
\hline FGF21 (pg/mL) & $379.2 \pm 327.4$ & $502.8 \pm 425.1$ & $546.8 \pm 385.6$ & 0.026 & 0.766 \\
\hline
\end{tabular}

All data are presented as the mean \pm SD.

PreDM, prediabetes patient; DM, diabetes patient; BMI, body mass index; SBP, systolic blood pressure; HOMA- $\beta$, homeostasis model assessment- $\beta$ cell function; HOMA-IR, homeostasis model-insulin resistance; HbAlc, glycated hemoglobin; NA, not applicable.

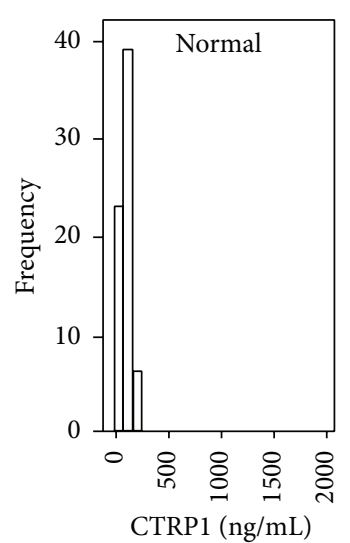

CTRP1 (ng/mL)

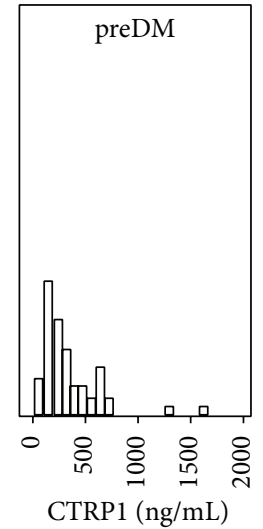

(a)

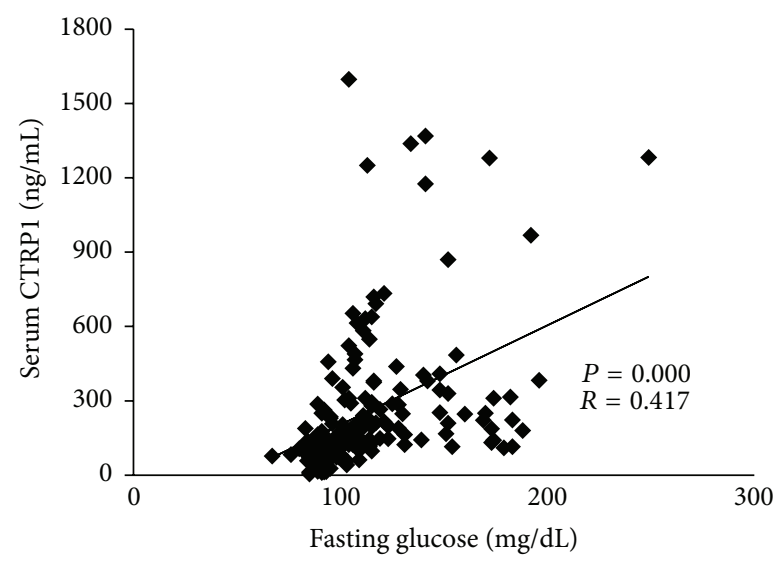

(c)

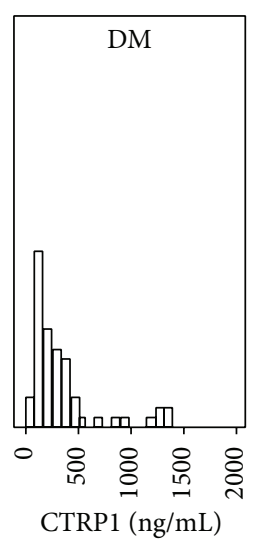

CTRP1 (ng/mL)

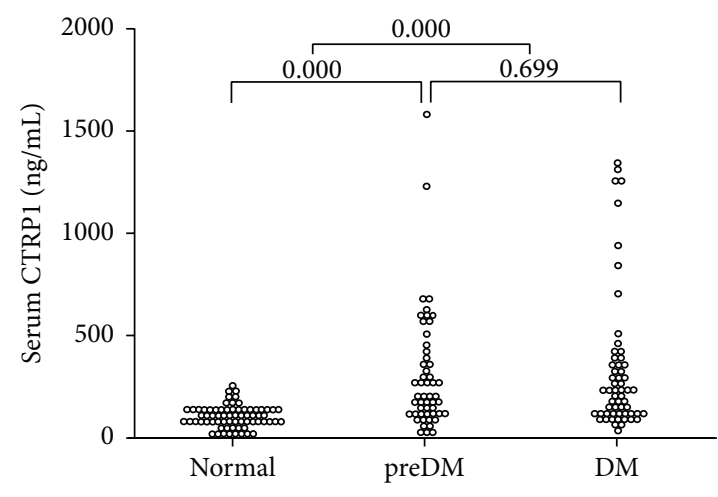

(b)

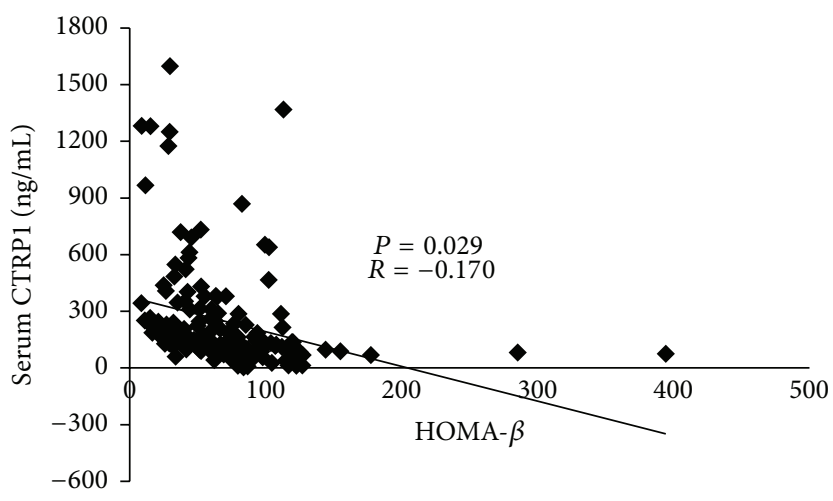

(d)

FIgURE 1: Circulating CTRP1 levels in healthy subjects and subjects with DM. Circulating CTRP1 levels were measured in three groups: normal, preDM, and DM patients. (a) Frequencies and distributions of circulating CTRP1 levels in three groups. (b) Circulating CTRP1 levels and statistical significance within three groups. Correlation analyses between circulating CTRP1 and fasting blood glucose levels (c) and HOMA- $\beta$ (d). preDM, prediabetes mellitus; DM, diabetes mellitus; HOMA- $\beta$, homeostasis model assessment- $\beta$. 


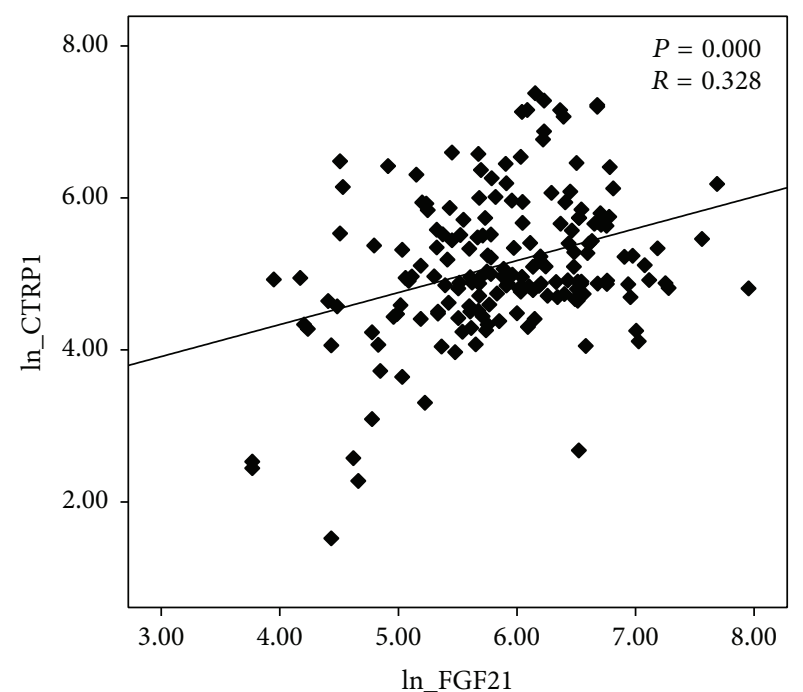

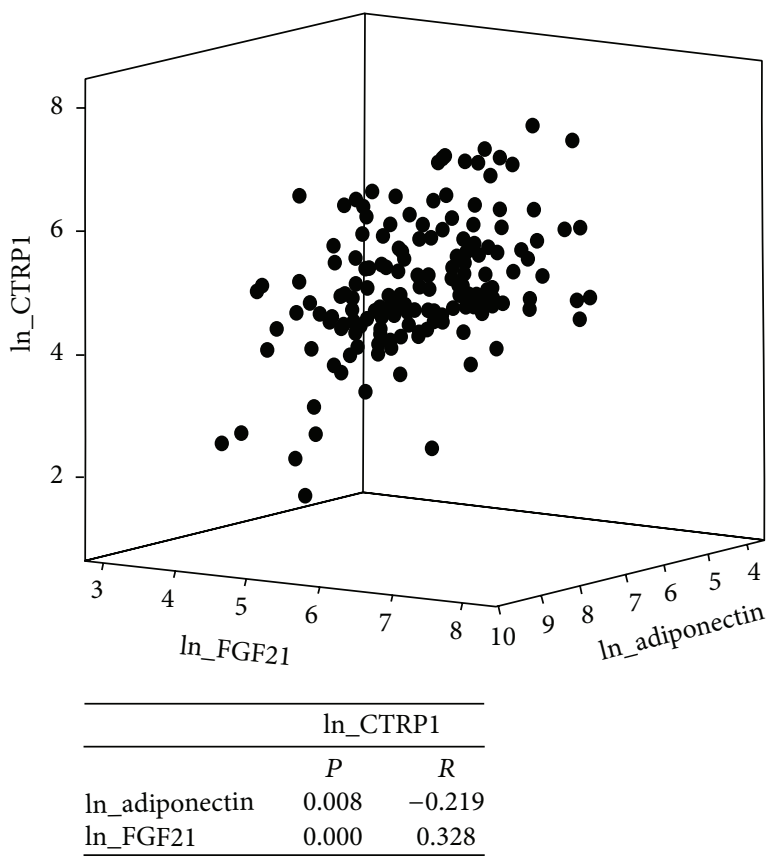

(b)

FIGURE 2: Correlation between circulating CTRP1 and DM-related cytokines. Circulating levels of CTRP1, FGF21, and adiponectin were measured in human sera. (a) Correlation analyses between circulating levels of CTRP1 and FGF21 in sera. (b) Correlation analyses between circulating levels of CTRP1, FGF21, and adiponectin in sera are showed in 3-dimensional dot plot (upper) and correlation coefficient (bottom). Correlations between indicated groups were analyzed using a generalized linear model. ln, natural logarithm.

TABLE 2: Correlation between circulating CTRP1 and fasting blood glucose levels.

\begin{tabular}{lcr}
\hline & \multicolumn{1}{c}{$\mathrm{CTRP1}^{\dagger}$} & $P$ value \\
\hline Glucose & 0.310 & 0.000 \\
\hline${ }^{\dagger}$ Ln transformed. BMI, age, sex, and medications were controlled variables \\
and medications included are SU, glinide, AGI, DPP-IV inhibitor, insulin, \\
ACE inhibitor, ARB, CCB, thiazide, $\beta$-blocker, statin, fenofibrate, cilostazol, \\
and thioctic acid.
\end{tabular}

TABLE 3: Pearson's correlation coefficient of CTRP1 with HOMA- $\beta$ and HOMA-IR.

\begin{tabular}{lcc}
\hline & & CTRP1 \\
& Coefficient & $P$ value \\
\hline HOMA- $\beta$ & -0.170 & 0.029 \\
HOMA-IR & 0.030 & 0.706 \\
\hline
\end{tabular}

HOMA- $\beta$, homeostasis model assessment- $\beta$ cell function; HOMA-IR, homeostasis model-insulin resistance.

Interestingly, it was revealed that circulating CTRP1 levels were positively correlated with circulating FGF21 levels. Multivariate analysis revealed that fasting blood glucose and TG levels showed an independent relationship with circulating CTRP1 levels. Circulating FGF21 and adiponectin levels also showed a strong correlation with circulating CTRP1 levels (Table 5). Furthermore, when the parameters were additionally adjusted for age, sex, BMI, and medications,
TABLE 4: Pearson's correlation coefficient between circulating CTRP1 levels and clinical data in univariate analysis.

\begin{tabular}{lcc}
\hline & Coefficient & $P$ value \\
\hline Sex & NA & 0.904 \\
BMI & 0.196 & 0.009 \\
SBP & 0.125 & 0.145 \\
DBP & 0.154 & 0.072 \\
Glucose & 0.455 & 0.000 \\
Total cholesterol & -0.180 & 0.018 \\
HDL cholesterol & -0.124 & 0.106 \\
LDL cholesterol & -0.145 & 0.061 \\
TGs & 0.167 & 0.030 \\
Adiponectin & -0.365 & 0.000 \\
FGF21 & 0.341 & 0.000 \\
\hline
\end{tabular}

BMI, body mass index; SBP, systolic blood pressure; DBP, diastolic blood pressure; HDL, high-density lipoprotein; LDL, low-density lipoprotein; TGs, triglyceride.

circulating CTRP1 levels were negatively correlated with circulating adiponectin levels and positively correlated with circulating FGF21 levels (Table 6, Figure 2(a)), whereas circulating FGF21 and adiponectin levels showed a negative correlation (Figure 2(b)). Because CTRP1 production is increased by TNF- $\alpha$ and IL- $1 \beta$ in adipose tissue $[8,20]$, we next assessed the relationship between circulating CTRP1 and other inflammatory cytokines (Table 7). Unexpectedly, 
TABLE 5: Correlation between circulating CTRP1 levels and clinical data by multivariate analysis.

\begin{tabular}{lccc}
\hline & Unstandardized & $\beta \pm$ SE & Standardized $\beta$ coefficient \\
\hline Sex & $-0.008 \pm 0.157$ & -0.012 & 1.000 \\
BMI & $-0.014 \pm 0.028$ & -0.046 & 0.626 \\
SBP $^{\dagger}$ & $-0.266 \pm 0.685$ & -0.034 & 0.699 \\
Glucose $^{\dagger}$ & $1.826 \pm 0.604$ & 0.420 & 0.003 \\
Total cholesterol & $0.002 \pm 0.002$ & 0.101 & 0.310 \\
TGs $^{\dagger}$ & $-0.301 \pm 0.166$ & -0.174 & 0.073 \\
Adiponectin $^{\dagger}$ & $-0.089 \pm 0.114$ & -0.081 & 0.435 \\
FGF21 $^{\dagger}$ & $0.309 \pm 0.094$ & 0.261 & 0.001 \\
Medication & & & Each variable is NS \\
Model & $R^{2}(0.423)$ & & 0.001 \\
\hline
\end{tabular}

${ }^{\dagger}$ Ln transformed. Medications included are SU, glinide, AGI, DPP-IV inhibitor, insulin, ACE inhibitor, ARB, CCB, thiazide, $\beta$-blocker, statin, fenofibrate, cilostazol, and thioctic acid. SE, standard error; BMI, body mass index; SBP, systolic blood pressure; TGs, triglycerides; NS, not significant.

TABLE 6: Kendall's tau-b correlation between circulating levels of CTRP1, adiponectin, and FGF21.

\begin{tabular}{lcc}
\hline & Adiponectin $^{\dagger}$ & FGF21 $^{\dagger}$ \\
\hline Correlation coefficient & -0.219 & 0.328 \\
$P$ value & 0.008 & 0.000 \\
\hline
\end{tabular}

${ }^{\dagger}$ Ln transformed. Medications included are SU, glinide, AGI, DPP-IV inhibitor, insulin, $\mathrm{ACE}$ inhibitor, $\mathrm{ARB}, \mathrm{CCB}$, thiazide, $\beta$-blocker, statin, fenofibrate, cilostazol, and thioctic acid.

no significant correlation was observed between circulating CTRP1 levels and other inflammatory cytokines.

\section{Discussion}

Previously, Xin et al. showed that circulating CTRP1 levels are elevated in Chinese subjects with T2DM and independently correlated with T2DM risk factors including BMI, fasting blood glucose levels, HbA1c, LDL cholesterol, and TNF- $\alpha$ [12]. In this study, circulating CTRP1 levels were positively and independently correlated with circulating FGF21 levels as well as BMI and fasting blood glucose levels in Korean subjects. In addition, we also show that circulating CTRP1 levels were significantly increased in subjects with preDM compared to healthy subjects, suggesting that CTRP1 is a prospective marker to prediagnose T2DM. Therefore, further investigation is necessary to reveal how CTRP1 affects impairment of glucose metabolism in the early stages of DM. At least, CTRP1 may affect insulin secretion because circulating CTRP1 levels were significantly associated with HOMA- $\beta$.

The previous studies reported that the administration of recombinant CTRP1 decreases blood glucose levels through activating the AMPK pathway and facilitating glucose uptake in the skeletal muscles in mice and that CTRP1 transgenic mice show an improved metabolic index against a high-fat diet [10]. Regardless of these beneficial effects of CTRP1 on glucose metabolism, subjects with T2DM show increased circulating CTRP1 levels compared to healthy subjects. Similar to CTRP1, circulating FGF21 levels in subjects with
T2DM were also unexpectedly higher compared to those of healthy subjects, although the administration of recombinant FGF21 in obese and diabetic rodent models ameliorates hyperglycemia and dyslipidemia by the enhancement of insulin sensitivity and glucose uptake [21]. These paradoxical results can be explained by the fact that a hyperglycemic state increases the compensatory secretion of CTRP1 and FGF21 [12]. However, the increased CTRP1 and FGF21 in subjects with T2DM fail to restore glucose metabolism. This can be explained by a possibility that abnormal physiological condition in T2DM may diminish the abilities of CTRP1 and FGF21 to enhance glucose metabolism. There is a report to support this possibility. Zhang et al. showed that metabolic imbalance in T2DM causes the FGF21 resistance [22]. Thus, whether CTRP1 also shows resistance in T2DM may be worth studying.

In the point of view that both circulating CTRP1 and FGF21 levels are increased in subjects with T2DM, the functional relationship between CTRP1 and FGF21 would be expected. CTRP1 and FGF21 have similar beneficial effects on glucose metabolism and they also share extracellular signalregulated kinase 1/2 (ERK1/2) and AMPK as downstream signal molecules [10, 23-25]. Thus, it is possible that CTRP1 and FGF21 are related with each other in balancing glucose homeostasis. However, further functional studies should be conducted to determine whether the relationship between CTRP1 and FGF21 is parallel, vertical, or synergistic in glucose metabolism.

There were some limitations in our study. First, we did not perform OGTT in healthy subjects. Although their fasting blood glucose levels were less than $100 \mathrm{mg} / \mathrm{dL}$, they could possibly be classified in the impaired glucose tolerance group if they performed accordingly on the OGTT. Second, we did not have HbAlc information from healthy subjects because we did not acquire samples to evaluate this measure. Third, subjects with T2DM treated as patients at the clinic were taking antidiabetic medications. Although the adjustment for medications was performed, cytokine and HOMA values might already have been altered by the therapeutic effect. 
TABLE 7: Kendall's tau-b correlation between circulating levels of CTRP1 and other cytokines under normal conditions.

\begin{tabular}{lccccccc}
\hline & FGF21 & RBP4 & IL-6 & MCP1 & TNF- $\alpha$ & Visfatin & AFABP \\
\hline Correlation coefficient & 0.199 & -0.026 & -0.059 & 0.051 & -0.028 & -0.012 & 0.037 \\
$P$ value & 0.017 & 0.755 & 0.478 & 0.539 & 0.744 & 0.896 & 0.660 \\
\hline
\end{tabular}

FGF21, fibroblast growth factor 21; RBP4, retinol-binding protein 4; IL-6, interleukin-6; MCP1, monocyte chemoattractant protein 1; TNF- $\alpha$, tumor necrosis factor- $\alpha$; AFABP, adipocyte fatty acid-binding protein.

In conclusion, this study shows the relationship between CTRP1 and FGF21. Further studies will shed light on how CTRP1 and/or FGF21 affect physiological function in subjects with T2DM.

\section{Disclosure}

Sora Han and Jong Dai Kim should be considered as co-first authors.

\section{Competing Interests}

The authors have no competing interests to declare.

\section{Authors' Contributions}

Sora Han and Jong Dai Kim contributed equally to the work reported and Young Yang and Won-Young Lee contributed equally to the work reported.

\section{Acknowledgments}

This research was supported by the Bio \& Medical Technology Development Program of the NRF, funded by the Korean government (MSIP2015M3A9B6027818), and Korea Health Industry Development Institute, funded by the Ministry of Health and Welfare (B110053-1101-0000200).

\section{References}

[1] B. Antuna-Puente, B. Feve, S. Fellahi, and J.-P. Bastard, "Adipokines: the missing link between insulin resistance and obesity," Diabetes \& Metabolism, vol. 34, no. 1, pp. 2-11, 2008.

[2] R. Meshkani and K. Adeli, "Hepatic insulin resistance, metabolic syndrome and cardiovascular disease," Clinical Biochemistry, vol. 42, no. 13-14, pp. 1331-1346, 2009.

[3] C. Manrique and J. R. Sowers, "Insulin resistance and skeletal muscle vasculature: significance, assessment and therapeutic modulators," Cardiorenal Medicine, vol. 4, no. 3-4, pp. 244-256, 2014.

[4] E. Z. Fisman and A. Tenenbaum, "Adiponectin: a manifold therapeutic target for metabolic syndrome, diabetes, and coronary disease?" Cardiovascular Diabetology, vol. 13, article 103, 2014.

[5] J.-H. Kim, K.-H. Bae, Y.-K. Choi et al., "Fibroblast growth factor 21 analogue LY2405319 lowers blood glucose in streptozotocininduced insulin-deficient diabetic mice by restoring brown adipose tissue function," Diabetes, Obesity and Metabolism, vol. 17, no. 2, pp. 161-169, 2015.

[6] J. Zhang and Y. Li, "Fibroblast growth factor 21, the endocrine FGF pathway and novel treatments for metabolic syndrome," Drug Discovery Today, vol. 19, no. 5, pp. 579-589, 2014.
[7] M. M. Seldin, S. Y. Tan, and G. W. Wong, "Metabolic function of the CTRP family of hormones," Reviews in Endocrine and Metabolic Disorders, vol. 15, no. 2, pp. 111-123, 2014.

[8] A. Schäffler and C. Buechler, "CTRP family: linking immunity to metabolism," Trends in Endocrinology and Metabolism, vol. 23, no. 4, pp. 194-204, 2012.

[9] G. W. Wong, S. A. Krawczyk, C. Kitidis-Mitrokostas, T. Revett, R. Gimeno, and H. F. Lodish, "Molecular, biochemical and functional characterizations of Clq/TNF family members: adiposetissue-selective expression patterns, regulation by PPAR- $\gamma$ agonist, cysteine-mediated oligomerizations, combinatorial associations and metabolic functions," Biochemical Journal, vol. 416, no. 2, pp. 161-177, 2008.

[10] J. M. Peterson, S. Aja, Z. Wei, and G. W. Wong, "CTRP1 protein enhances fatty acid oxidation via AMP-activated protein kinase (AMPK) activation and acetyl-CoA carboxylase (ACC) inhibition," The Journal of Biological Chemistry, vol. 287, no. 2, pp. 1576-1587, 2012.

[11] Y. Shen, L. Lu, Z. H. Liu et al., "Increased serum level of CTRP1 is associated with low coronary collateralization in stable angina patients with chronic total occlusion," International Journal of Cardiology, vol. 174, no. 1, pp. 203-206, 2014.

[12] Y. Xin, X. Lyu, C. Wang et al., "Elevated circulating levels of CTRP1, a novel adipokine, in diabetic patients," Endocrine Journal, vol. 61, no. 9, pp. 841-847, 2014.

[13] P. Shabani, H. N. Khaledi, M. Beigy et al., "Circulating level of CTRP1 in patients with nonalcoholic fatty liver disease (NAFLD): is it through insulin resistance?" PLoS ONE, vol. 10, no. 3, article e0118650, 2015.

[14] X. Pan, T. Lu, F. Wu et al., "Circulating complement-Clq TNFrelated protein 1 levels are increased in patients with type 2 diabetes and are associated with insulin sensitivity in Chinese subjects," PLoS ONE, vol. 9, no. 5, Article ID e94478, 2014.

[15] L. Chalupova, A. Zakovska, and K. Adamcova, "Development of a novel enzyme-linked immunosorbent assay (ELISA) for measurement of serum CTRP1: a pilot study: measurement of serum CTRP1 in healthy donors and patients with metabolic syndrome," Clinical Biochemistry, vol. 46, no. 1-2, pp. 73-78, 2013.

[16] K. E. Davis and P. E. Scherer, "Adiponectin: no longer the lone soul in the fight against insulin resistance?" Biochemical Journal, vol. 416, no. 2, pp. e7-e9, 2008.

[17] American Diabetes Association, "Standards of medical care in diabetes-2010," Diabetes Care, vol. 33, supplement 1, pp. S11S61, 2010.

[18] S. E. Park, E.-J. Rhee, W.-Y. Lee et al., "The role of serum adipocyte fatty acid-binding protein on the development of metabolic syndrome is independent of pro-inflammatory cytokines," Nutrition, Metabolism and Cardiovascular Diseases, vol. 22, no. 6, pp. 525-532, 2012.

[19] D. R. Matthews, J. P. Hosker, A. S. Rudenski, B. A. Naylor, D. F. Treacher, and R. C. Turner, "Homeostasis model assessment: 
insulin resistance and $\beta$-cell function from fasting plasma glucose and insulin concentrations in man," Diabetologia, vol. 28, no. 7, pp. 412-419, 1985.

[20] K.-Y. Kim, H. Y. Kim, J. H. Kim et al., “Tumor necrosis factor$\alpha$ and interleukin- $1 \beta$ increases CTRP1 expression in adipose tissue," FEBS Letters, vol. 580, no. 16, pp. 3953-3960, 2006.

[21] P. Iglesias, R. Selgas, S. Romero, and J. J. Díez, "Biological role, clinical significance, and therapeutic possibilities of the recently discovered metabolic hormone fibroblastic growth factor 21," European Journal of Endocrinology, vol. 167, no. 3, pp. 301-309, 2012.

[22] X. Zhang, D. C. Y. Yeung, M. Karpisek et al., "Serum FGF21 levels are increased in obesity and are independently associated with the metabolic syndrome in humans," Diabetes, vol. 57, no. 5, pp. 1246-1253, 2008.

[23] H. Akiyama, M. Otani, S. Sato et al., "A novel adipokine Clq/TNF-related protein 1 (CTRP1) regulates chondrocyte proliferation and maturation through the ERK1/2 signaling pathway," Molecular and Cellular Endocrinology, vol. 369, no. 12, pp. 63-71, 2013.

[24] B. Emanuelli, S. G. Vienberg, G. Smyth et al., "Interplay between FGF21 and insulin action in the liver regulates metabolism," Journal of Clinical Investigation, vol. 124, no. 2, pp. 515-527, 2014.

[25] X. Ge, C. Chen, X. Hui, Y. Wang, K. S. L. Lam, and A. Xu, "Fibroblast growth factor 21 induces glucose transporter-1 expression through activation of the serum response factor/Ets-like protein-1 in adipocytes," The Journal of Biological Chemistry, vol. 286, no. 40, pp. 34533-34541, 2011. 


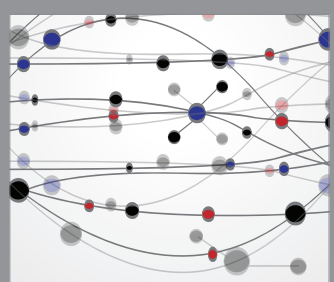

The Scientific World Journal
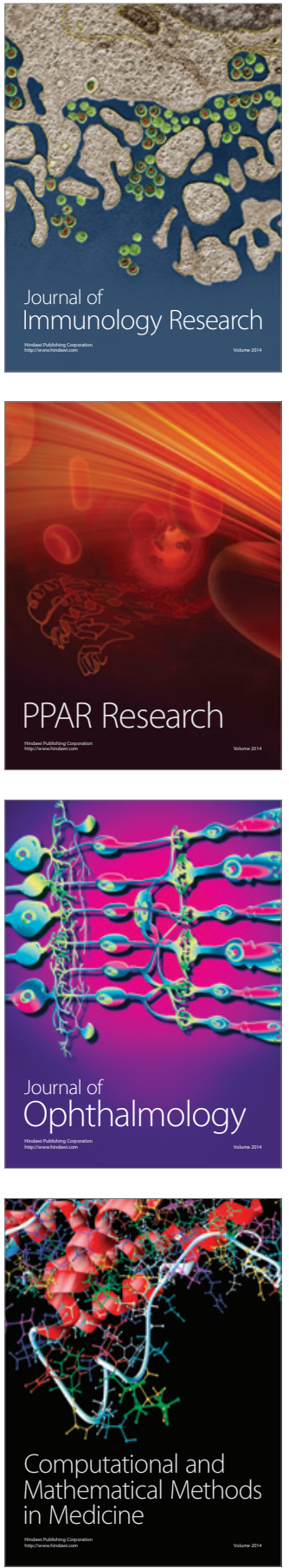

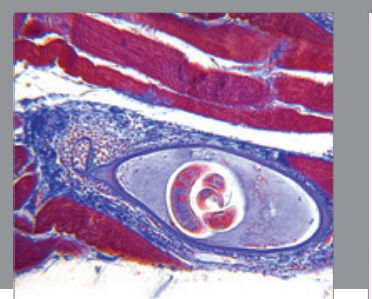

Gastroenterology Research and Practice

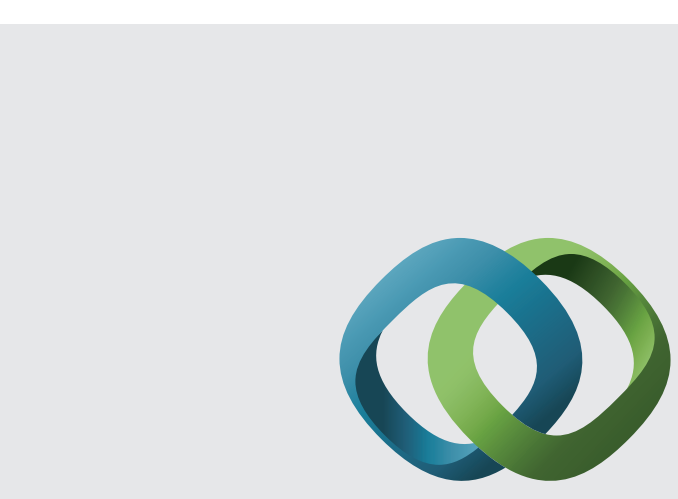

\section{Hindawi}

Submit your manuscripts at

http://www.hindawi.com
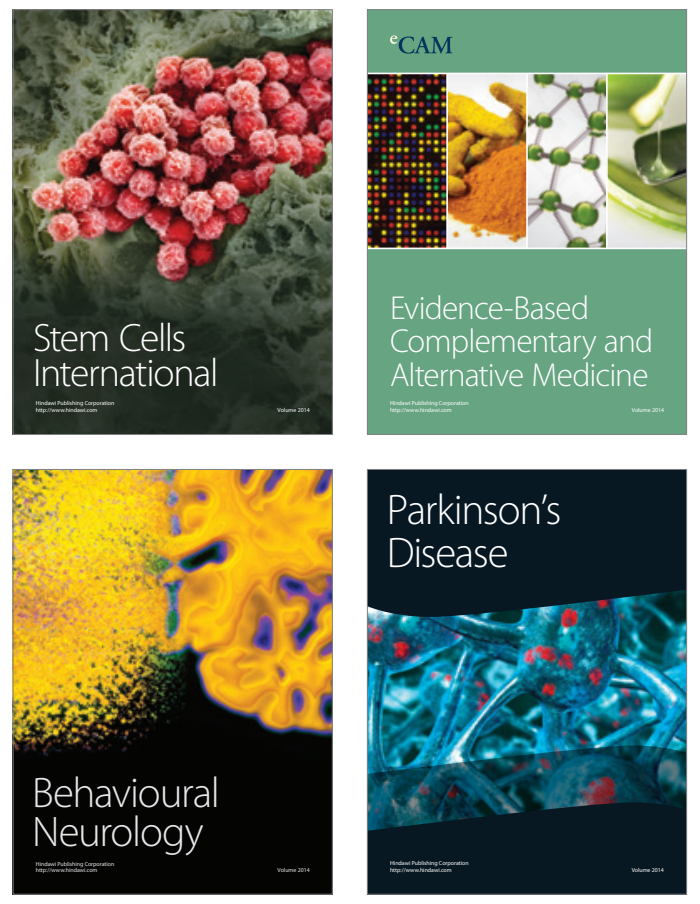
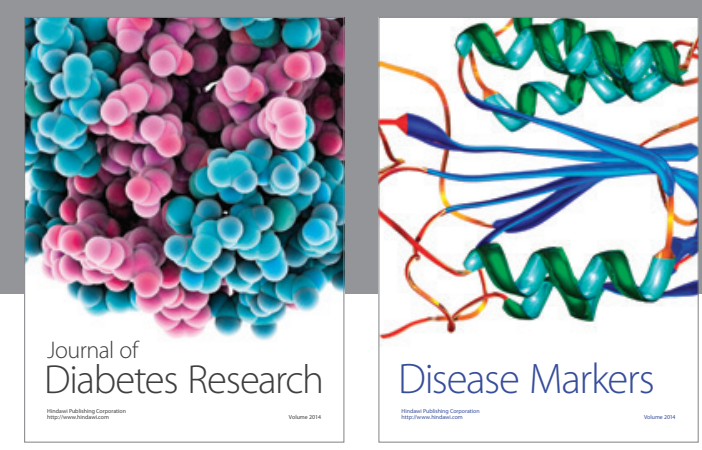

Disease Markers
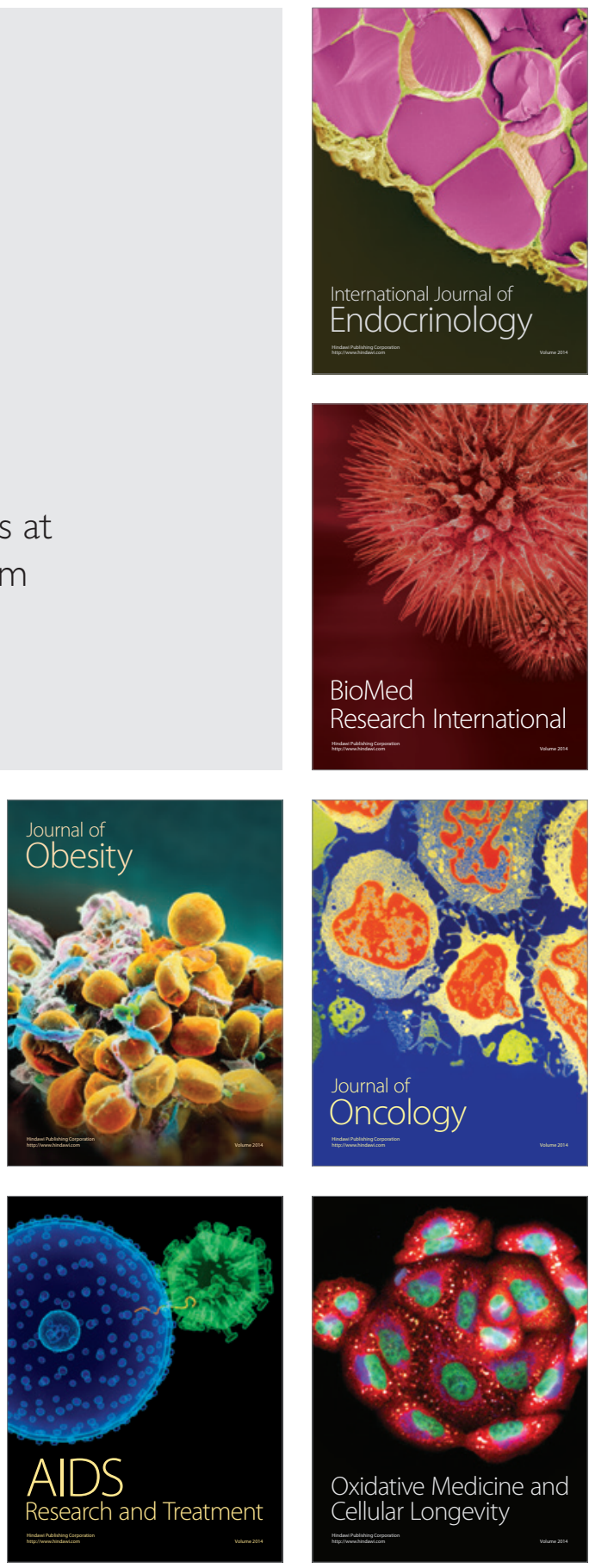